\title{
Forest Fire Monitoring Based on Mixed Wireless Sensor Networks
}

\author{
Teng $\mathrm{Ma}^{1}$, Yun Liu ${ }^{1} *$, Junsong $\mathrm{Fu}^{1}$ and Ya Jing ${ }^{2}$ \\ 1. School of Electronic and Information Engineering, Key Laboratory of \\ Communication and Information Systems, Beijing Municipal Commission of \\ Education, Beijing Jiaotong University, Beijing, 100044, China \\ 2. State Grid Jibei Electric Power CO.LTD. Material Branch (North China Power \\ Equipment \& Material General Corp.), State Grid, Beijing, 100075, China \\ ${ }^{1}$ \{08111003, liuyun*, 12120067\}@bjtu.edu.cn \\ jiing.ya@nc.sgcc.com.cn
}

\begin{abstract}
Forest fires can be fatal threats. Motivated by the need to detect fire early and locate forest fires clearly, in this paper, we propose a paradigm called the forest fire monitoring paradigm (FFMP). The purpose of the FFMP is for forest fire early detection and locating based on mixed wireless sensor networks (WSNs). Different from pure static and mobile WSNs, mixed WSNs are composed of both mobile sensor nodes and static sensor nodes. Mixed WSNs are a tradeoff between cost and coverage. In the FFMP, the mobile sensor nodes perform as cluster heads and they will construct a backbone network in which the mobile sensor nodes can connect with their neighbors and be capable of transmitting data to the base station. Each static sensor node chooses one neighboring mobile sensor node as its cluster head and uploads the generated messages to the cluster head. The mobile sensor nodes then fuses the information and transfers the fusion results to the base station, where the data were further processed to obtain the temperature distribution graph and locate the fires. The simulation illustrates that our approach performs well in early forest fire detection and locating. In addition, our approach can significantly prolong the lifetime of WSNs.
\end{abstract}

Keywords: mixed wireless sensor networks, forest fire detection, information fusion, Delaunay triangulation

\section{Introduction}

Forest fires can be fatal threats. Over 13000 forest fires occurred in China between 1950 and 2014. In a few of these fires, large areas of forest were destroyed, and in some fires, people or other animals died. Consequently, forest fire monitoring has been widely researched in many fields [1-4].

Satellite based monitoring is a popular method used to detect forest fires. However, the long scanning period and low resolution of the satellites $[1,2]$ restricts the effectiveness of satellite-based forest fire detection. Moreover, satellites usually cannot forecast forest fires before the fire has spread uncontrollably.

In the recent years, advances in microprocessors, radio techniques, memory and micro-sensors enable wireless sensor networks (WSNs), has been used to gather process and transmit information. The improvements of sensor nodes' capabilities make distributed micro-sensing, an activity in which a set of sensor nodes collaborates with each other to achieve a huge sensing task, possible. WSNs have been successfully used in many applications, such as intrusion detection [3] and environment monitoring [4]. There is no 
doubt that we can employ WSNs to monitor forest fires; as such, various paradigms have been proposed. We will give a detailed presentation of the approaches in section 2 . However, most of the approaches monitor the fire roughly and cannot provide a clear boundary of the fire.

Motivated by the need to clearly detect fires and predict the developing trend of the fire, in this paper, a WSN paradigm, the forest fire monitoring paradigm (FFMP), is proposed. The goals of designing such a paradigm are presented as follows:

- Forest fire early detection.

- Build a temperature distribution graph.

- Locate the fire clearly.

- Prolong the lifetime of WSN as far as possible.

In FFMP, two kinds of sensor nodes are deployed in our interest area. The number of the static sensor nodes should be large enough to cover the entire area. Because of the mobility of the mobile sensor node, the number of mobile sensor nodes can be moderate. In the initial phase of FFMP, the base station needs to calculate the proper location of each mobile sensor node and send location messages to the mobile sensor nodes. After moving to the proper location, the mobile sensor nodes perform as cluster heads. It is hoped that they will construct a backbone network in which the mobile sensor nodes can connect with their neighbors and be capable of transmitting data to the base station.

Each static sensor node chooses one neighboring mobile sensor node as its cluster head. As a result, several clusters are formatted. In the cluster formation phase, we employ an algorithm in [5]. After deciding which cluster head to follow, the static sensor nodes can upload the readings to the cluster head periodically. The mobile sensor node then fuses the information and transmits the fusion results to the base station, where the data are further processed to build the temperature distribution graph and predict the fire's developing trend. The base station is the bridge between the networks and the users. The information about the boundary and the developing trend of the fire is transmitted to the users, who take appropriate measures based on the information.

We apply the theory of the bipartite graph to the matching of mobile sensor nodes and their proper locations. The bipartite graph is a common graph structure in the theory of graphs. The properties of bipartite graphs have been researched for a long time. In our early work [6] on moving strategies for mobile sensor nodes in mixed WSNs, we proposed an algorithm to match the bipartite graph with a 2-exchange optimization algorithm. A better moving strategy was proposed in [7]; hence, we used that strategy in this paper.

A location sensitive information fusion mechanism is proposed. When a fire accident happens in a forest, the temperature is strongly related to the location. If two sensor nodes are far from each other, it makes no sense to fuse their data. As such, the users need to preset a distance parameter to decide whether the data generated by the two sensors should be fused. If the sources of the data are close enough, we need to fuse both the locations and their temperatures.

We also provide a novel definition of fire location. We divide the interest areas into many square grids, where the lengths of the sides are preset. The smaller the grids, the more accurate the fire location we can get. The max set of grids with a center with temperatures beyond a preset threshold is defined as the location of the fire. A challenging problem is how to calculate the temperature of each grid. We solve the problem by introducing the Delaunay Triangulation and estimate the center temperature of a grid by the corresponding triangle's three vertexes. In addition, we develop an algorithm to predict the developing trend of the fire.

The remainder of this paper is organized as follows. Section 2 describes some of the 
related work. We present the forest fire monitoring paradigm in Section 3. In Section 4, we show the simulation results and, at last, we conclude this paper in Section 5.

\section{Related Work}

We categorize the existing related work into three classes: forest fire monitoring, in-network data processing and mobile sensor nodes moving strategies in mixed WSNs.

a) Mobile sensor nodes moving strategies in mixed sensor networks

To our knowledge, the authors in [8] first focused on deploying a mixture of static and mobile sensors to meet the coverage requirements and proposed a bidding protocol in mixed sensor networks. In the bidding protocol, static nodes are treated as bidders or consumers and mobile nodes are treated as service providers. The mobile sensor nodes choose coverage holes to heal based on the bid. However, applying the bidding protocols in [8], several sensors may have to move a long distance, which would take more time to complete the construction of the sensor network.

The authors in [9] introduced an improved bidding protocol called the proxy-based bidding protocol. To reduce the distances that the mobile sensors had to move, the proxy-based bidding protocol proposes that mobile sensors perform virtual movements from small holes to large holes and that they only perform physical movements after the final destinations have been identified.

b) In-network data processing

As discussed previously, the energy of sensor nodes in WSNs is strictly limited. In-network data processing is an effective approach to use to reduce energy consumption. In this paper, we focus on one of the in-network data processing techniques, the data fusion technique. To date, various types of information fusion algorithms have been proposed, such as probability theory-based information fusion algorithms, the Dempster-Sharfer (D-S) evidence theory-based information fusion algorithms, and the fuzzy set theory-based information fusion algorithms.

c) Forest fire monitoring

Traditional forest fire detection systems relied on satellite imagery [10-11]. Recently, WSNs have been widely used in forest fire monitoring systems [2, 12]. In [2], the authors combined the WSNs with neural networks and described data collecting and processing for real-time forest fire detection. The authors in [12] modeled the forest fire detection problem as a k-coverage problem in WSNs. In addition, they presented a simple data aggregation scheme based on the FWI System. This data aggregation scheme significantly prolongs the network lifetime, because it only delivers the data that is of interest to the application.

\section{Forest Fire Monitoring Paradigm}

In this section, we describe our forest fire monitoring paradigm in detail. For the development of FFMP, we made some assumptions about the static and mobile sensor nodes.

The static sensor nodes are densely deployed in the forest in order to obtain a high sensing coverage rate. They are strictly limited by energy, computation, communication band and storage. Even though it is not necessary to densely deploy the mobile sensor nodes, their quality must be god enough that they can cover the interest area by their communication capability.

The energy of a mobile sensor node is much more than that of a static node. We also assume that the mobile sensor nodes are capable of moving and avoiding obstacles which can guarantee that the mobile sensor nodes can move to a proper location.

All the sensor nodes can obtain its geographic information by equipment such as GPS or 
some other form of localization techniques [13-14]. This is essential, because when monitoring forest fires, we not only want to know whether there is fire, but also where is the fire. Therefore, the sensors should capable of locating themselves.

The paradigm is composed of three phases: (1) mobile sensor nodes deployment, (2) information fusion, and (3) locating the fire.

\subsection{Mobile Sensor Node Deployment and Cluster Formation}

After being randomly scattered in the forest, the sensor nodes need to automatically organize themselves into a network. In this paper, we organize our network in a hierarchical way. Our network is divided into two layers: the static sensor nodes compose the lower layer and the mobile sensor nodes compose the higher layer. The responsibility of the lower layer is monitoring the environment and uploading the information to the higher layer. The sensor nodes in the higher layer need to process the data and upload the processed data to the base station. Initially, the static sensor nodes turn into an energy-saving sleep mode and keep receiving message modules, but only when they are live. The mobile sensors first upload their location information to the base station through a routing protocol, such as a flooding routing protocol. To prolong the lifetime and balance the load of the WSNs, we divide the interest area into regular hexagons, like the cellular systems in Figure 1. At each core of the cellular, a mobile sensor node exists and controls the static sensor nodes located in the entire cellular.

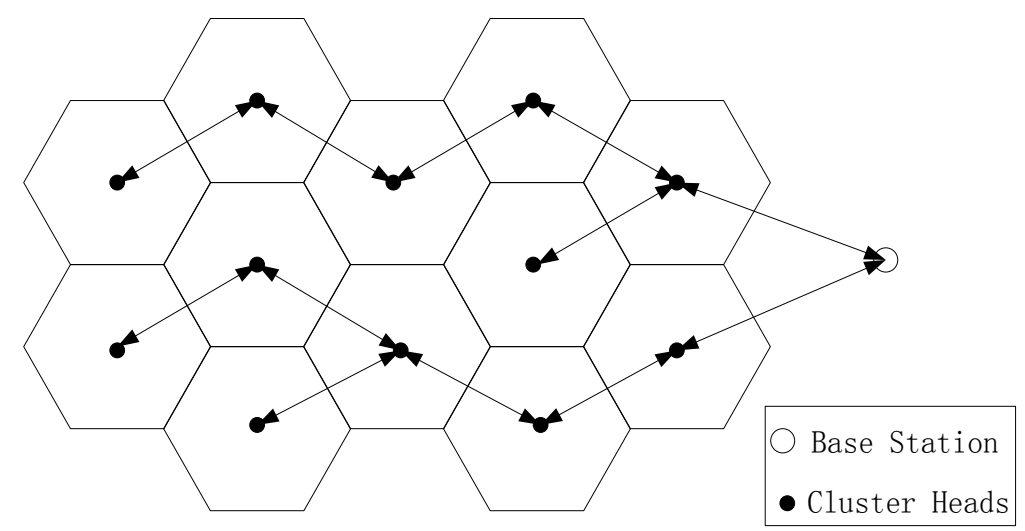

Figure 1. Deployment of the Mobile Sensor Nodes

The cores of the cellular system are calculated by the base station in a centralized way. Haven collected the location information of the mobile sensor nodes and calculating the cores of the cellular system, the base station needs to match the sensor nodes and the cores. A possible goal of the match is making the total moving distance as short as possible. Several relational problems have been researched.

A distributed protocol, called the bidding protocol, is proposed in [15] to assist in the movement of the mobile sensors. In the protocol, static sensors detect coverage holes locally by using Voronoi diagrams, and bid for mobile sensors based on the size of the detected hole. Mobile sensors choose coverage holes to cover based on the bid. Though the simulation results illustrate that our algorithm provides suitable tradeoffs between the coverage and sensor costs, the total moving distance of all of the mobile sensors is too long and, as a result, in [16], the authors proposed a better algorithm named deployment protocols with virtual movement.

To further decrease the total moving distance, as our previous work in [6], we discussed how to match mobile sensors with coverage holes in order to obtain a shorter moving distance 
in a centralized way. In [7], the authors improved our approach. In this paper, we employ the method in [7] to match the mobile sensor nodes and the cores of the cellular system. Figure 2 illustrates a good match based on [7].

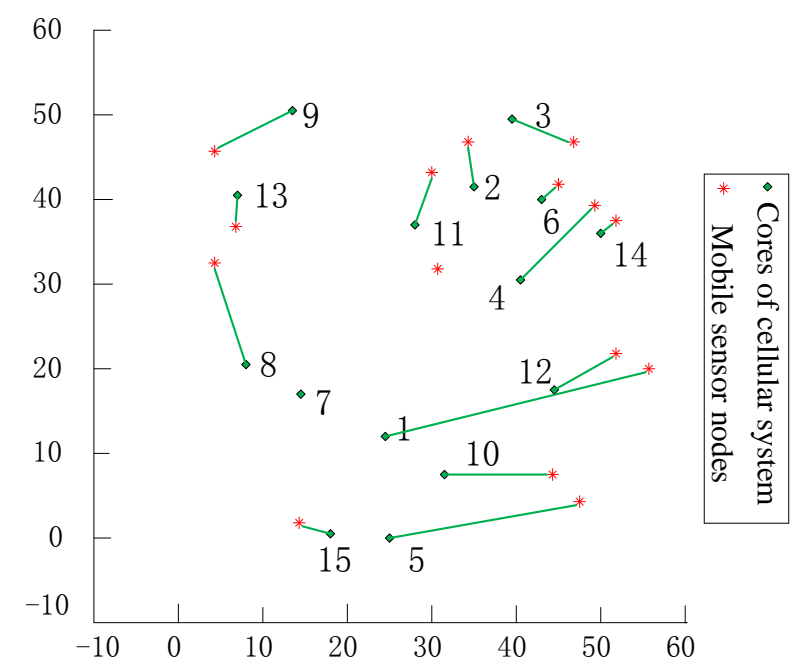

Figure 2. Matches between the Mobile Sensor Nodes and the Cores of the Cellular System

The base station broadcasts a match message in the network and each mobile sensor node moves to its destination based on the match message. The mobile sensor serves as cluster heads in the network; they must let all the static sensor nodes in the WSN know that. To do this, each mobile sensor node broadcasts a simple advertisement message, shown of the form [header, ID]. The header distinguished the message as an announcement message and the ID is used to identify the mobile sensor node.

Each static sensor determines its cluster head by choosing the nearest one. A classic method in [14] can be employed to solve this problem. Each sensor node chooses the cluster head that requires the minimum communication energy based on the received signal strength. After each sensor node has decided its cluster head, it must inform the cluster head that it will be a member of the cluster and upload its monitoring data to its cluster head periodically. The cluster head has the responsibility to fuse the data received from its cluster and upload the data fusion results to the base station through a multi-hop manner.

\subsection{Information Fusion Phase}

The responsibilities of the mobile sensor nodes in our paradigm are for controlling the clusters and processing and transmitting the data uploaded by the static sensor nodes. However, the mobile sensor nodes don't monitor the surrounding environment. Opposed to the mobile sensor nodes, the static sensor nodes need to collect the information.

In this paper, we use temperature to indicate the forest fire. The structure of the message generated by the static sensor nodes is [location, temperature]. The temperature is used to detect the fire and location is used to locate the fire. As discussed previously, wireless sensor nodes are strictly limited by some resources, such as energy and communication bandwidth. Therefore, saving energy is one of the most important issues in WSNs. Recognizing that in-network computations would generate less energy consumption than that of communication; we can save the energy by fusing the data and transmitting the data fusion 
results, instead of the original data. The loss of information is the cost of information fusion. When building the temperature distribution graph, the more information we have, the more accurate the graph is.

In conclusion, there is a tradeoff between effectiveness and efficiency. In most situations, each sensor node has a limited communication bandwidth. Without the loss of generality, we assume that each mobile sensor node can transmit the data of $\mathrm{K}$ pieces of information successfully in a constant period. If the quantity of information generated by the static sensor nodes the threshold, say $\mathrm{K}$, the mobile sensor node needs to compress the information into $\mathrm{K}$ pieces, while containing the important information as much as possible.

We define the importance of a message generated by a static sensor node $n_{i}$ as $I_{i}$, which is significantly affected by the position. The further a sensor node is from its neighbors, the more important the message is. As an example in Figure 3, intuitively, the black circle is much more important than the blue and red circles, because the black circle has no neighbor near enough and can't be replaced by its near neighbors. However, the red and blue circles can be replaced by each other, and as a result, they are not as important as the black one. In this paper, we assume that $I_{i} \propto d_{1}$, where $d_{1}$ is the distance between $n_{i}$ and its nearest neighbor.

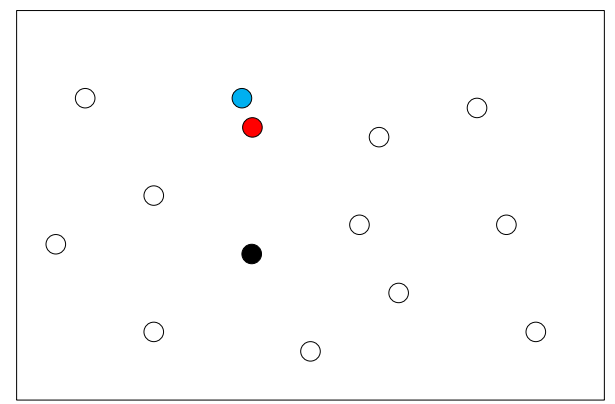

Figure 3. The Importance of a Message

We provide a formal problem statement as follows:

Given $\mathrm{N}$ pieces of information of the form [location, temperature], we need to fuse the information into $\mathrm{K}$ pieces, while retaining the important information as much as possible.

A possible approach is randomly selecting $\mathrm{K}$ pieces of information and transmitting them to the base station; however, this is not a good choice because some valuable messages would be deleted. We should select $\mathrm{K}$ pieces of representative information. For convenience, we present each piece of information by a circle whose location is decided by the location contained in the information. Figure 3 illustrates an example, where each circle represents a piece of information and its location is location contained in the information. If $\mathrm{K}=13$, we need to delete one circle and, intuitively, the red circle is the one that should be deleted, because the red circle is too close to another circle. Let $d_{i}$ be the distance of a circle $c$ to its ith nearest neighbor, then we should delete $c_{i}$, with the smallest $d_{1}$. When two or more circles have the same $d_{1}$, the circle with the smaller $d_{2}$ should be deleted, and so on. In Figure 3 , the $d_{1}$ of the red and blue circles are the same with each other; hence, the red circle is deleted because $d_{2}$ of the red circle is smaller than that of the blue circle. 


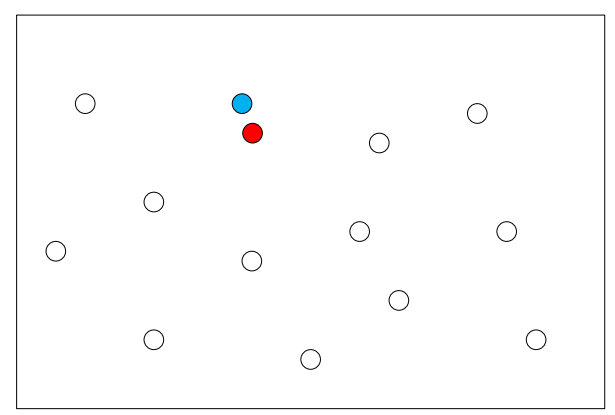

Figure 4. Representative Information

If the deleted circle is very close to one that has not been deleted, the information is redundant. To improve the accuracy of the monitoring information, we can make full use of all of the information and fuse the deleted information with its nearest neighbor. We need to preset a parameter $\mathrm{d}$. The deleted data should be fused with its neighbor, when the distance between them is smaller than d. Fusing the location of the two pieces of the message is simple. Assume that the location $_{1}$ is $\left(\mathrm{x}_{1}, \mathrm{y}_{1}\right)$ and location $_{2}$ is $\left(\mathrm{x}_{2}, \mathrm{y}_{2}\right)$, then the location is fused by location $_{\mathrm{f}}=\left(\frac{\mathrm{x}_{1}+\mathrm{x}_{2}}{2}, \frac{\mathrm{y}_{1}+\mathrm{y}_{2}}{2}\right)$.

The fusing temperature is much more complex. Consequently, in this paper, we employ the probability based information fusion theory. Information fusion has been widely researched and applied [17]. There are several types of information fusion, including probability based information fusion, Dempster-Shafer based information fusion and rough set theory based information fusion.

Probability theory is able to deal effectively with uncertainty problems, like the random measurement errors of the sensor node. Probability based information fusion algorithms are suitable for low-level information fusion. Bayesian data fusion enables the fusion of pieces of data and lies at the core of the probability based data fusion algorithms. We assume a state-space representation $\mathrm{X}$, where the Bayesian data fusion algorithm provides a method for computing the posterior conditional probability distribution/density of the hypothetical state $\mathrm{x}_{\mathrm{k}}$, given the set of measurements $\mathrm{Z}=\left\{\mathrm{z}_{1}, \mathrm{z}_{2}, \ldots, \mathrm{z}_{\mathrm{n}}\right\}$, where $\mathrm{n}$ is the number of sensor nodes whose readings need to be fused and the prior distributions, as follows:

$$
\mathrm{p}\left(\mathrm{x}_{\mathrm{k}} \mid \mathrm{Z}\right)=\frac{\mathrm{p}\left(\mathrm{x}_{\mathrm{k}}\right) * \mathrm{p}\left(\mathrm{Z} \mid \mathrm{x}_{\mathrm{k}}\right)}{\sum \mathrm{p}(\mathrm{Z})}
$$

where: $\mathrm{p}\left(\mathrm{Z} \mid \mathrm{x}_{\mathrm{k}}\right)$ is called the likelihood function and is based on the given sensor measurement model and $\sum \mathrm{p}\left(\mathrm{Z} \mid \mathrm{x}_{\mathrm{k}}\right)$ is a constant and is merely a normalization term to ensure that the probability density function is integrated to one. We maximize $p\left(x_{k} \mid Z\right)$ to obtain $\mathrm{x}_{\mathrm{k}}$, which is the data fusion result. In our approach, $\mathrm{Z}$ is the set of temperatures that needs to be fused and $\mathrm{p}\left(\mathrm{Z} \mid \mathrm{x}_{\mathrm{k}}\right)$ is decided by the property of the sensor nodes. In addition, we set $\mathrm{p}\left(\mathrm{x}_{\mathrm{k}}\right)$ to a uniform distribution in the range of possible temperatures.

In this paper, we preset a threshold $\mathrm{T}_{\mathrm{d}}$, which is used to decide whether the deleted message can be fused. If two message locations are very far from each other, the real physical temperature of the two locations is very different and it makes no sense to fuse the two pieces of the message. Therefore, if $d_{1}$ of the deleted message is smaller than $T_{d}$, we fuse the deleted message with its nearest neighbor.

\subsection{Fire Locating}

After receiving all of the temperature data from the interest area, the base station needs to locate the fire and predict the developing trend. The location of the fire is defined as follows: 
Definition of LOCATION of fire: The LOCATION of the fire is the max set of Grids where the centers' temperature is beyond a preset threshold. The resolution $\mathrm{R}$ of the Grids is also preset by the users.

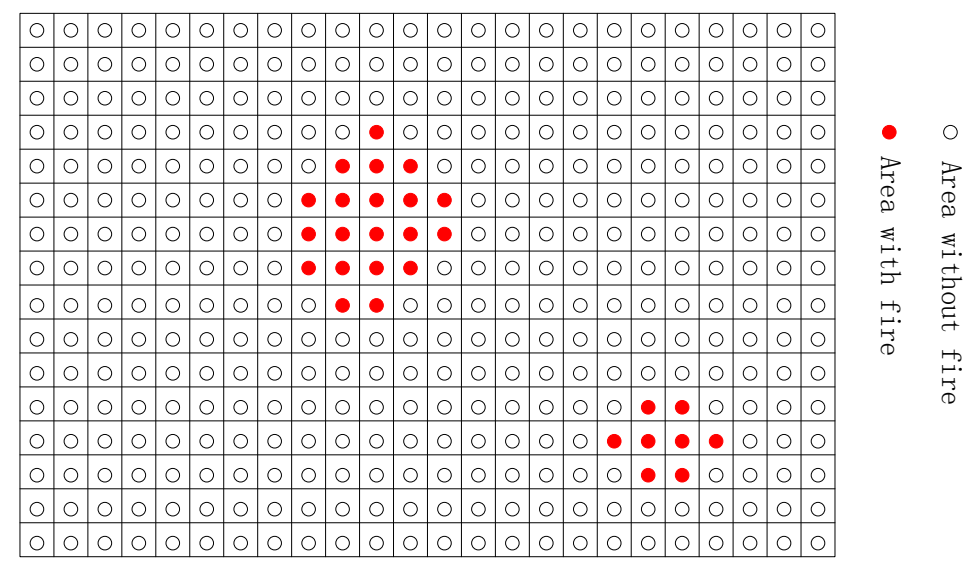

Figure 5. LOCATION of the Fire

As an example in Figure 5, the resolution of the Grids is set to be $\mathrm{d}^{2}$ (i.e., the distance of the two grids' center is d). The smaller the $d$ is, the more accurate the result we get. In Figure 5, there are two fire incidents which are represented by the red areas. A challenge is calculating each grid center's temperature, based on the monitoring data. The locations of the data are discrete and, in our approach, we achieve a continuous model by Delaunay triangulation. The Delaunay triangulation and the Voronoi diagram are dual constructions.

Given a set of two-dimensional nodes, we used a randomized incremental algorithm to triangulate them into many triangles [6]. In this way, we can obtain any point's temperature. Consider a point located at $\left(\mathrm{x}_{\mathrm{i}}, \mathrm{y}_{\mathrm{i}}\right)$ that is covered by a triangle with three vertices $\left(\mathrm{x}_{\mathrm{i} 1}, \mathrm{y}_{\mathrm{i} 1}\right)$, $\left(\mathrm{x}_{\mathrm{i} 2}, \mathrm{y}_{\mathrm{i} 2}\right),\left(\mathrm{x}_{\mathrm{i} 3}, \mathrm{y}_{\mathrm{i} 3}\right)$. The temperature in $\left(\mathrm{x}_{\mathrm{i} 1}, \mathrm{y}_{\mathrm{i} 1}\right),\left(\mathrm{x}_{\mathrm{i} 2}, \mathrm{y}_{\mathrm{i} 2}\right)$ and $\left(\mathrm{x}_{\mathrm{i} 3}, \mathrm{y}_{\mathrm{i} 3}\right)$ is $\mathrm{T}_{\mathrm{i} 1}, \mathrm{~T}_{\mathrm{i} 2}$ and $\mathrm{T}_{\mathrm{i} 3}$ , respectively. Then a plane is determined by the three points of $\left(x_{i 1}, y_{i 1}, T_{i 1}\right),\left(x_{i 2}, y_{i 2}, T_{i 2}\right)$, $\left(\mathrm{x}_{\mathrm{i} 3}, \mathrm{y}_{\mathrm{i} 3}, \mathrm{~T}_{\mathrm{i} 3}\right)$ and we can obtain the plane as follows:

We assume the plane is defined as $\mathrm{A} * \mathrm{x}+\mathrm{B} * \mathrm{y}+\mathrm{C} * \mathrm{~T}+\mathrm{Z}=0$. Then we put the three points into the equation and get:

$$
\left\{\begin{array}{l}
A * x_{11}+B * y_{i 1}+C * T_{i 1}+Z=0 \\
A * x_{i 2}+B * y_{i 2}+C * T_{i 2}+Z=0 \\
A * x_{i 3}+B * y_{i 3}+C * T_{i 3}+Z=0
\end{array}\right.
$$

We can obtain the temperature at $(\mathrm{x}, \mathrm{y})$ by $\mathrm{T}=-\mathrm{A} * \mathrm{x}-\mathrm{B} * \mathrm{y}-\mathrm{Z}$, where:

$$
\begin{gathered}
A=\frac{\left(\frac{T_{i 1} * x_{i 2}-T_{i 1} * x_{i 3}+T_{i 2} * *_{i 3}-T_{i 2} * *_{i 1}+T_{i 3} * *_{i 1}-T_{i 3} * x_{i 2}}{y_{i 1} *_{i 3}-y_{i 1} * x_{i 2}-y_{i 2} * x_{i 3}+y_{i 2} * x_{i 1}-y_{i 3} * x_{11}+y_{i 3} * x_{i 2}}\right)\left(y_{i 2}-y_{i 1}\right)+\left(T_{i 2}-T_{i 1}\right)}{x_{i 1}-x_{i 2}}, \\
B=\frac{T_{i 1} * x_{i 2}-T_{i 1} * x_{i 3}+T_{i 2} * x_{i 3}-T_{i 2} * x_{i 1}+T_{i 3} * x_{i 1}-T_{i 3} * x_{i 2}}{y_{i 1} * x_{i 3}-y_{i 1} * x_{i 2}-y_{i 2} * x_{i 3}+y_{i 2} * x_{i 1}-y_{i 3} * x_{i 1}+y_{i 3} * x_{i 2}}, \\
Z=-A * x_{i 1}-B * y_{i 1}-T_{i 1} .
\end{gathered}
$$

Figure 6 illustrates the continuous model. We can obtain each location's temperature by equation (5). Then, we can locate the fire, which is defined previously. 


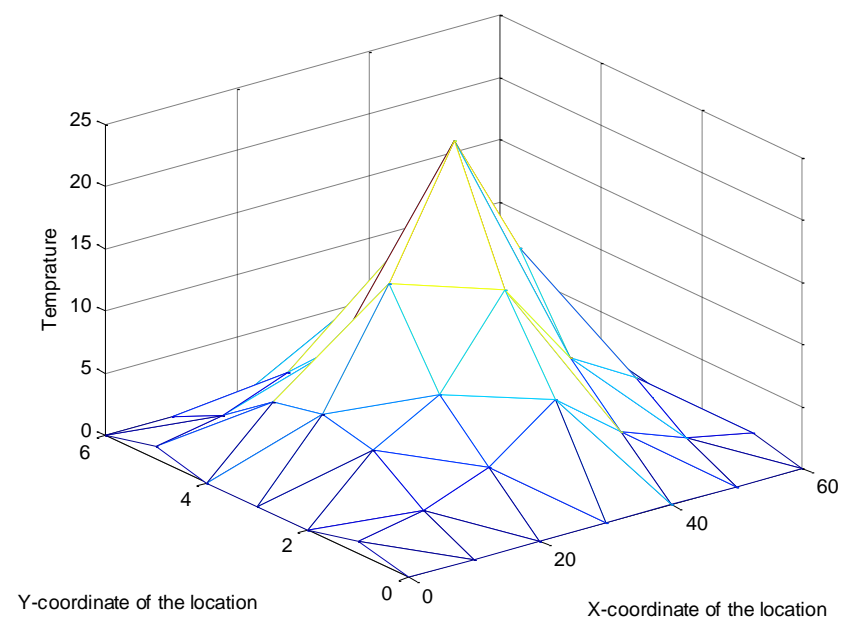

Figure 6. An Example of a Continuous Model

In the ideal situation, when the users want to know the temperature information about the forest, all the monitoring data generated by the static sensor nodes are transferred to the base station at once and the base station constructs the continuous model, as shown in Figure 6, based on the data. However, because of the different surrounding environments, the location and the workload of the sensor nodes, the data flows into the base station as a sequence. Therefore, we need to process the data stream, rather than a static dataset, and use an incremental algorithm to construct the Delaunay triangulations. We can calculate the new triangulation based on the previous result; this is the core idea of the randomized incremental algorithm.

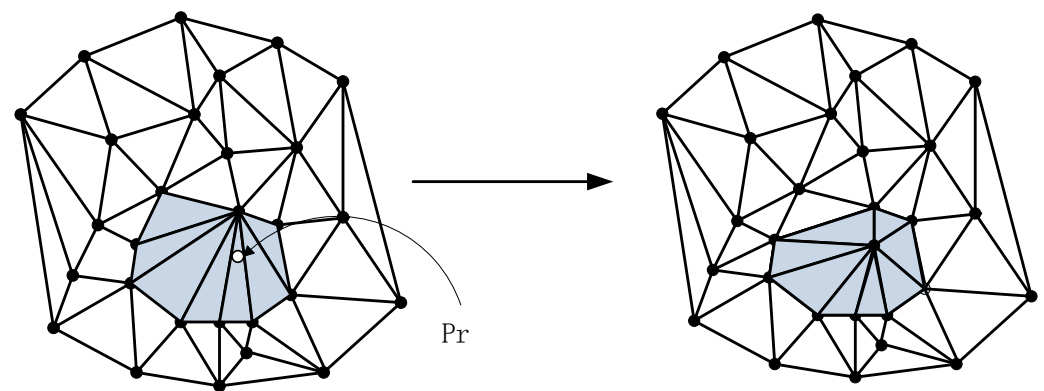

$\begin{array}{ll}\text { (a) Previous result before } p_{r} & \text { (b) Result after having added } p_{r}\end{array}$

Figure 7. Triangulation in the Randomized Incremental Algorithm

A triangulation is illustrated in Figure 7(a). When a new data point, $\mathrm{p}_{\mathrm{r}}$, is added into the dataset, we need to calculate the new triangulation. As shown in Figure 7(b), the new triangulation is only different from the previous triangulation in the shaded part. Therefore, we just have to calculate a part of the new triangulation, rather than calculate the entire triangulation again. Before we introduce the algorithm, the illegal edge is defined as follows (Figure 8): 


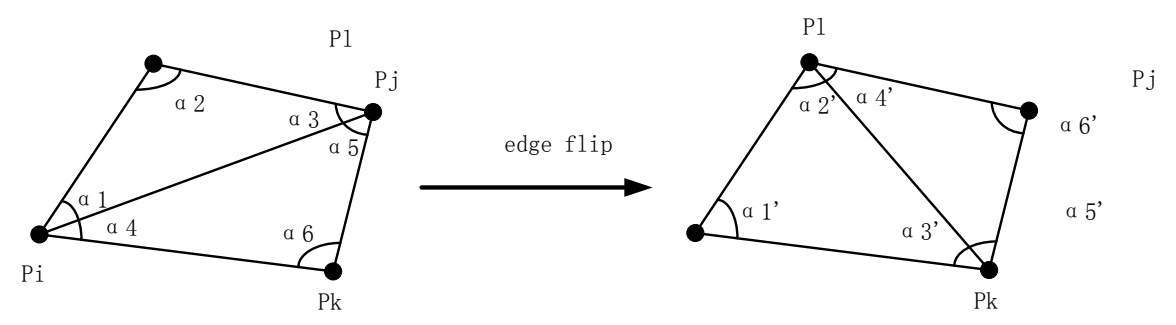

(a) $\mathrm{p}_{\mathrm{i}} \mathrm{p}_{\mathrm{j}}$ is the illegal edge

(b) No illegal edge

Figure 8. Edge Flip

In Figure 8(a), there are six angles: $\alpha_{1}, \alpha_{2}, \alpha_{3}, \alpha_{4}, \alpha_{5}$, and $\alpha_{6}$. There are also six angles in Figure 8(b): $\alpha_{1}^{\prime}, \alpha_{2}^{\prime}, \alpha_{3}^{\prime}, \alpha_{4}^{\prime}, \alpha_{5}^{\prime}$, and $\alpha_{6}^{\prime}$. If the $\min \left(\alpha_{\mathrm{i}}\right)<\min \left(\alpha_{\mathrm{i}}^{\prime}\right), 1 \leq \mathrm{i} \leq 6$, then $p_{i} p_{j}$ is an illegal edge.

We assumed that $\mathrm{T}$ is the triangulation and the randomized incremental algorithm is described as follows:

1. do (add $\mathrm{p}_{\mathrm{r}}$ to $\mathrm{T}$ )

2. find the triangle $p_{i} p_{j} p_{k} \in T$ that has $p_{r}$ in it

3. if $\left(p_{r}\right.$ is located in $\left.p_{i} p_{j} p_{k}\right)$

4. then line $p_{r}$ and the three vertexes of $p_{i} p_{j} p_{k}$, as illustrated in

Figure 4.6

5. LegalizeEdge $\left(p_{r}, p_{i} p_{j}, T\right)$;

6. LegalizeEdge $\left(p_{r}, \quad p_{j} p_{k}, T\right)$;

7. LegalizeEdge $\left(p_{r}, \quad p_{k} p_{i}, T\right)$;

8. else $\left(* p_{r}\right.$ is located on the edge, we assume that the edge is $\left.p_{i} p_{j}\right)$

9. line $p_{r}$ and $p_{k}, p_{l}$, as shown in Figure 4.7

10. LegalizeEdge $\left(p_{r}, \quad p_{i} p_{j}, T\right)$;

11. LegalizeEdge $\left(p_{r}, p_{j} p_{k}, T\right)$;

12. LegalizeEdge $\left(p_{r}, \quad p_{k} p_{j}, T\right)$;

13. LegalizeEdge $\left(p_{r}, p_{j} p_{i}, T\right)$;

14. return $(\mathrm{T})$;

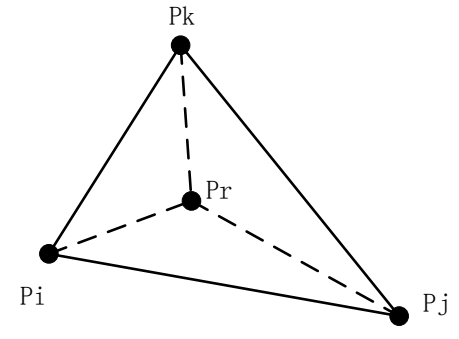

Figure 9. $p_{r}$ is Located in $p_{i} p_{j} p_{k}$

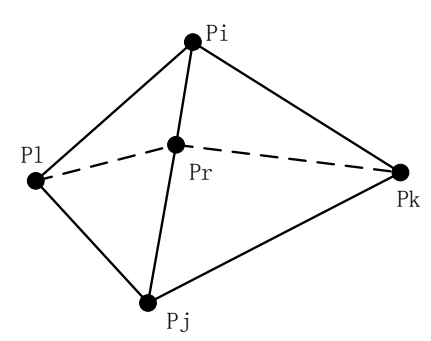

Figure 10. $p_{r}$ is Located on the Edge

The function of LegalizeEdge $\left(p_{r}, p_{i} p_{j}, T\right)$ is described as follows: 


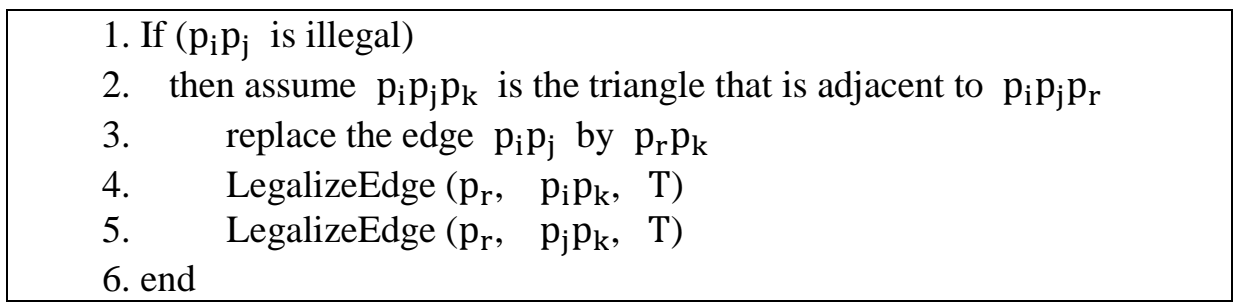

More detailed explanations of the algorithm can be found in [18]. Based on the incremental algorithm, we can construct the continuous temperature distribution graph and locate the fire at any time. With the increase in the receiving data, the base station can obtain a more accurate graph. Having obtained the temperature distribution graph, the base station checks each location's temperature and tells the user whether there is a fire in the forest. If there is no fire, a NORMAL message is sent to the users. On the other hand, if there is fire, an ABNORMAL message is sent to the users. In the meanwhile, the base station locates the fire and sends the LOCATION of the fire to the users which can be used by the users to put out the fire.

\section{Performance Evaluations}

We use ns-3 simulator [19] (version 3.21) to evaluate the performance of the FFMP approach. To simulate the readings of the sensor nodes, we extract 100 similar sub-traces from the temperature traces provided by the Live from Earth and Mars project [20] at the University of Washington. Each sub-trace contains 800 readings, as illustrated in Figure 11. To reduce the randomness, we do the same experiment 10 times and present the average result. For each experiment, we employ one or several sub-traces to simulate the readings of the sensor nodes. To simulate the spatial correlation of the sensor readings, we attempt to dispatch the successive readings in the sub-trace to the neighboring sensor nodes in the simulated network.

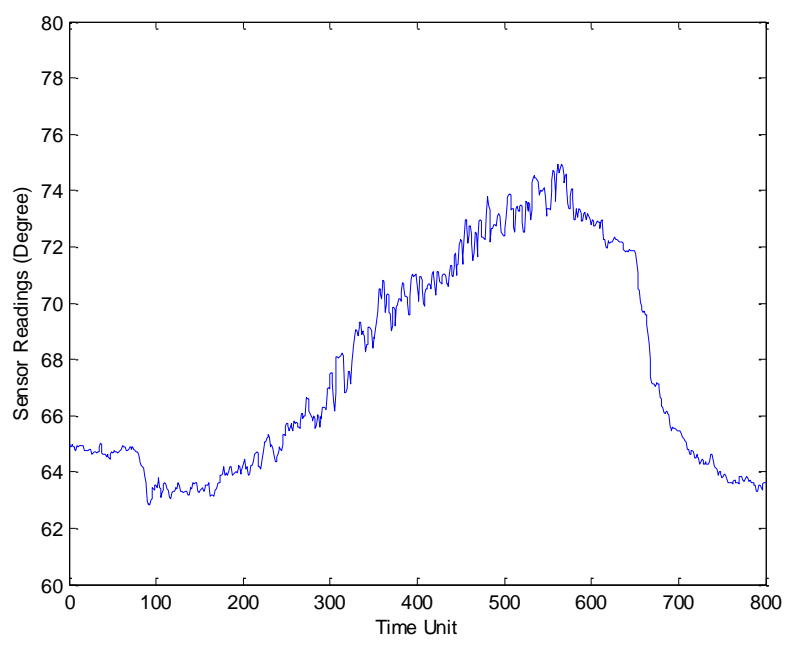

Figure 11. A Possible Sub-trace

We randomly deployed 250 static sensor nodes, 27 mobile sensor nodes and a base station in a $50 \mathrm{~m} * 42 \mathrm{~m}$ area. After the mobile sensor nodes moving to the proper locations, the 
deployment of the sensor nodes is shown in Figure 12.

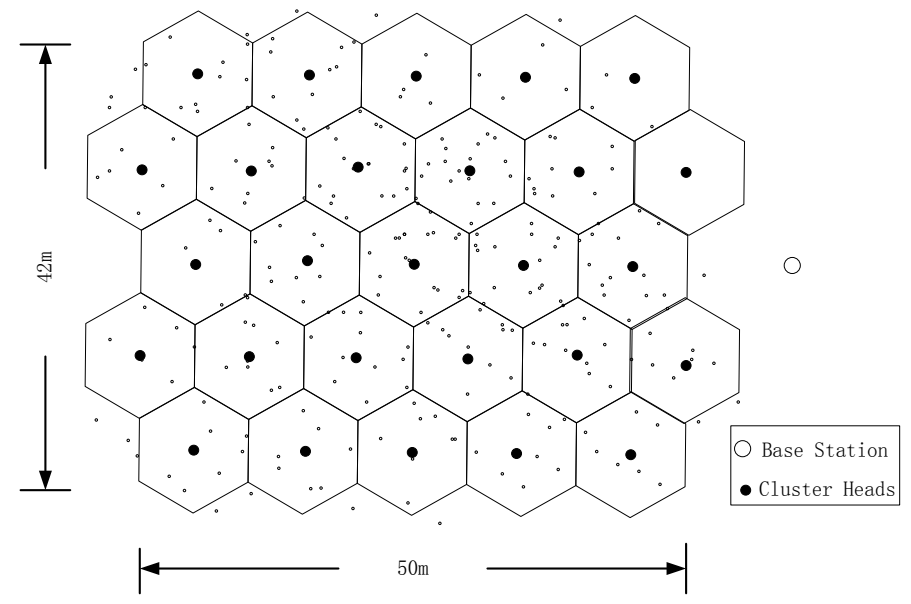

Figure 12. Deployment of the Senor Nodes

In the phase of the information fusion, we set $\mathrm{K}=5$ and $\mathrm{d}=2 \mathrm{~m}$. As a result, the base station receives about 125 pieces of messages and each piece of message contains a temperature and its location. The base station processes the data and builds a temperature distribution graph, as illustrated in Figure 13. In addition, we assume that if the area's temperature is beyond 70 degrees, there is a fire accident and the resolution $\mathrm{R}$ is set to $1 \mathrm{~m}$.

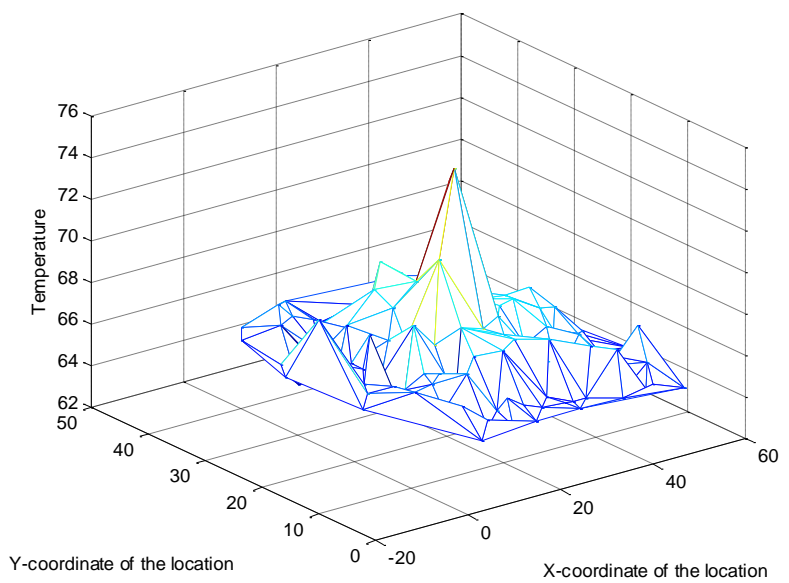

Figure 13. Temperature Distribution Graph

We can find that a fire accident happens in the interest area and the location of the fire can be calculated based on the definition of the LOCATION of the fire. The simulation result is shown in Figure 14. 


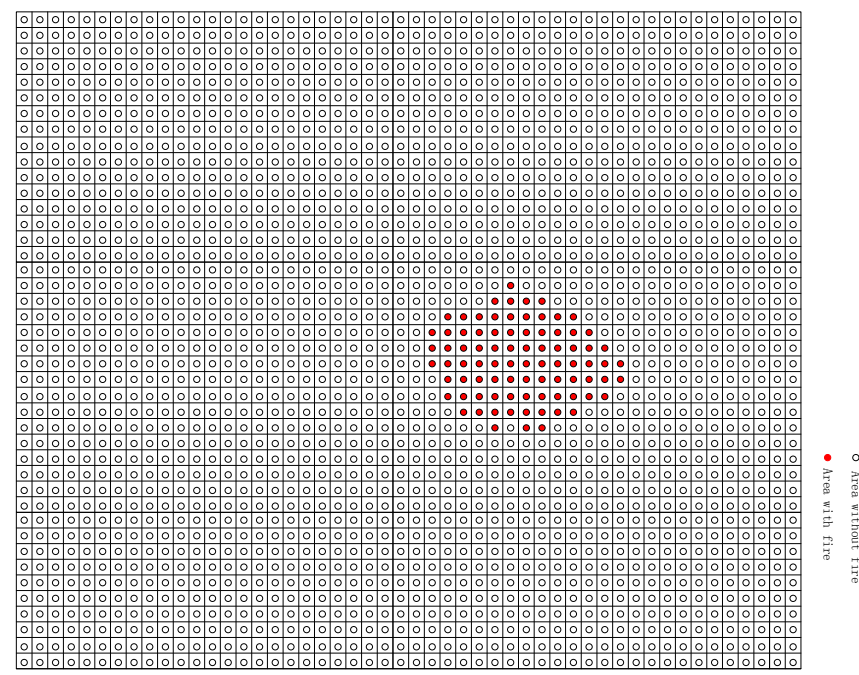

Figure 14. LOCATION of the Fire

We compare the number of live nodes, based on our paradigm, against the static WSN. The time unit is set to 10 seconds and in each time unit, the network collects the information about the forest fire once. We employ an 802.11 physical layer and a 1.0 Mb/s 802.11 MAC layer. Each sensor node has three modes: sending message, receiving message and sleeping. As in [21], the static sensor nodes take $60 \mathrm{~nJ}$ to send a bit of data and $50 \mathrm{~nJ}$ to receive a bit of data. When there is no need to communicate with the other nodes, the sensor node goes to sleep and takes $0.016 \mathrm{~mW}$ per second. A piece of message takes 20 bytes in our simulation. The initial energy of the static sensor nodes are set at 1 Joule. In addition, we assume that the mobile sensor nodes have much more energy than the static sensor nodes and that they are not dead until at least one static sensor node has been dead. In the static WSN, the base station employs the TAG routing tree to communicate with the sensor nodes. The simulation result is presented in Figure 15.

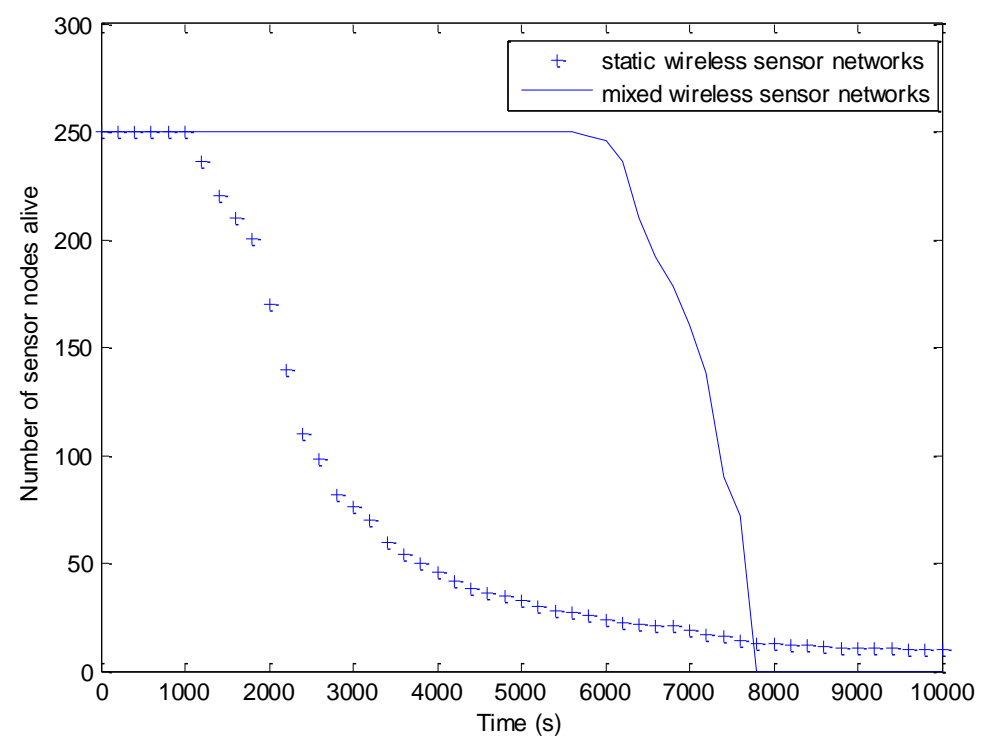

Figure 15. Number of Nodes Live Over Time 
As illustrated in Figure 15, the lifetime of the mixed WSN is much longer than the traditional static WSNs. This can be explained in mixed WSNs, where the mobile sensor nodes transmit most of the data and the static sensor nodes save most of the energy.

\section{Conclusions}

In this paper, a forest fire detection approach, called the FFMP, is proposed based on mixed WSNs. Different from the existing work focused on forest fire detection; we try to obtain more information about the fire. The goals of designing the paradigm include forest fire early detection, temperature distribution graph construction, and fire locating and prolonging the lifetime of the WSN as far as possible. To achieve these goals, our approach consisted of three phases. For each phase, we designed a series of rules. A series of simulation experiments were conducted to evaluate our approach and the simulation results showed that the FFMP performs very well in effectiveness, energy saving and accuracy. As our future work, we plan to design a more robust and intelligent mechanism for forest fire monitoring. Fire developing trend prediction based on a temperature distribution graph and the LOCATION of the fire is another challenge for us. In addition, reducing the data transmission and prolonging the life of the network further are another two important issues.

\section{Acknowledgements}

This research is supported by National Natural Science Foundation under Grant 61371071, Beijing Natural Science Foundation under Grant 4132057, Academic Discipline and Postgraduate Education Project of Beijing Municipal Commission of Education.

\section{References}

[1] Z. Li, S. Nadon and J. Cihlar, "Satellite-based detection of Canadian boreal forest fires: Development and application of the algorithm", International Journal of Remote Sensing, vol. 21, no. 16, (2000), pp. 3057-3069.

[2] L. Yu, N. Wang and X. Meng, "Real-time forest fire detection with wireless sensor networks", Wireless Communications, Networking and Mobile Computing, 2005. Proceedings. 2005 International Conference on IEEE, vol. 2, (2005), pp. 1214-1217.

[3] F. Bao, R. Chen, M. J. Chang, et al., "Hierarchical trust management for wireless sensor networks and its applications to trust-based routing and intrusion detection", Network and Service Management, IEEE Transactions on, vol. 9, no. 2, (2012), pp. 169-183.

[4] L. M. Oliveira and J. J. Rodrigues, "Wireless sensor networks: a survey on environmental monitoring", Journal of communications, vol. 6, no. 2, (2011), pp. 143-151.

[5] W. B. Heinzelman, A. P. Chandrakasan and H. Balakrishnan, "An application-specific protocol architecture for wireless microsensor networks", Wireless Communications, IEEE Transactions on, vol. 1, no. 4, (2002), pp. 660-670.

[6] Z. J. Zhang, J. S. Fu and H. C. Chao, "An energy-efficient motion strategy for mobile sensors in mixed wireless sensor networks", International Journal of Distributed Sensor Networks, (2013).

[7] Z. Q. Hao and Z. J. Zhang, "Two Centralized Energy-Efficient Deployment Algorithms for Mobile Nodes in a Mixed Wireless Sensor Network", Journal of Computers, vol. 24, no. 4, (2014), pp. 32-43.

[8] G. Wang, G. Cao and T. LaPorta, "A bidding protocol for deploying mobile sensors", Network Protocols, 2003. Proceedings. 11th IEEE International Conference on. IEEE, (2003), pp. 315-324.

[9] G. Wang, G. Cao, P. Berman, et al., "Bidding protocols for deploying mobile sensors", Mobile Computing, IEEE Transactions on, vol. 6, no. 5, (2007), pp. 563-576.

[10] Z. Wan, P. Wang and X. Li, "Using MODIS land surface temperature and normalized difference vegetation index products for monitoring drought in the southern Great Plains, USA", International Journal of Remote Sensing, vol. 25, no. 1, (2004), pp. 61-72.

[11] T. J. Lynham, C. W. Dull and A. Singh, "Requirements for space-based observations in fire management: A report by the wild land fire hazard team, committee on earth observation satellites (CEOS) disaster management support group (DMSG)", Geoscience and Remote Sensing Symposium, 2002. IGARSS'02. 2002 IEEE International. IEEE, vol. 2, (2002), pp. 762-764. 
[12] M. Hefeeda and M. Bagheri, "Wireless sensor networks for early detection of forest fires", Mobile Adhoc and Sensor Systems, 2007. MASS 2007. IEEE Internatonal Conference on. IEEE, (2007), pp. 1-6.

[13] L. Hu and D. Evans, "Localization for Mobile Sensor Networks," Proc. ACM MobiCom, (2004) September, pp. 45-57.

[14] K. F. Ssu, C. H. Ou and H. C. Jiau, "Localization with Mobile Anchor Points in Wireless Sensor Networks," IEEE Trans. Vehicular Technology, vol. 54, no. 3, (2005) May, pp. 1187-1197.

[15] G. Wang, G. Cao and T. LaPorta, "A bidding protocol for deploying mobile sensors", Network Protocols, 2003. Proceedings. 11th IEEE International Conference on. IEEE, (2003), pp. 315-324.

[16] G. Wang, G. Cao and T. La Porta, "Movement-assisted sensor deployment", Mobile Computing, IEEE Transactions on, vol. 5, no. 6, (2006), pp. 640-652.

[17] B. Khaleghi, A. Khamis, F. O. Karray, et al., "Multisensor data fusion: A review of the state-of-the-art", Information Fusion, vol. 14, no. 1, (2014), pp. 28-44.

[18] M. De Berg, M. V. Kreveld, M. Overmars, et al., "Computational geometry", Springer Berlin Heidelberg, (2000).

[19] http://www.nsnam.org/.

[20] "Live from Earth and Mars (LEM) Project", (2006), http://www-k12.atmos.washington.edu/k12/grayskies/.

[21] M. Wu, J. Xu, X. Tang, et al., "Top-k monitoring in wireless sensor networks", Knowledge and Data Engineering, IEEE Transactions on, vol. 19, no. 7, (2007), pp. 962-976.

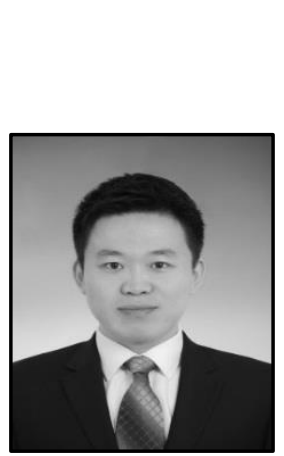

\section{Authors}

Teng Ma is currently pursuing the $\mathrm{PhD}$ degree with Department of Information and Communication Engineering in Beijing Jiaotong University. His research interests include wireless sensor network, Internet of Things and green networking.

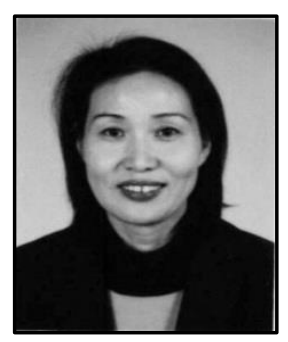

Yun Liu is a Professor of Communication and Information Systems, Beijing Jiaotong University; Dean of Communication Engineering Department, Beijing Jiaotong University; Director of key Laboratory of Communication and Information Systems, Beijing Municipal Commission of Education; Director of Institute of Network Consensus Security, Beijing Jiaotong University; Vice-chair of Teachers and Staff Representative Committee, Beijing Jiaotong University. She is currently a Fellow of IET, UKand specialist enjoying special government allowance. In addition, she is an evaluation expert of State Scientific and Technological reward, State Natural Sciences Fund in communication, National High Technology Research and Development Program (HTRDP), an advanced counselor of China Tietong Company, and an advanced counselor of Beijing Municipal Office of Internet Propaganda and Management.

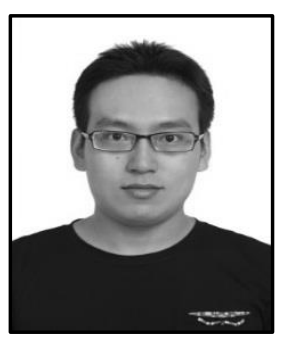

Jun-song Fu, received his bachelor's degree in communication engineering in 2012 from Beijing Jiaotong University, China. Currently, he is a doctoral student in Beijing Jiaotong University. He has been doing research in the field of wireless sensor networks, reputation systems, data fusion and data mining algorithms. 


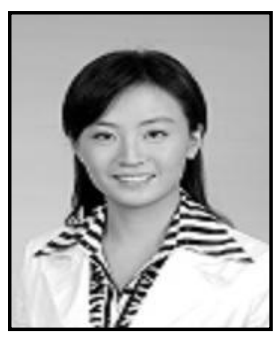

Ya Jing received her Master Degree in Communication and Information System in 2009 from Beijing Jiaotong University. She is in charge of enterprise data security\& informationization construction since 2009 inState Grid Jibei Electric Power CO.LTD. Material Branch (North China Power Equipment \& Material General Corp.), State Grid, Beijing. 